\title{
The perception of visual motion during movements of the eyes and of the head
}

\author{
MICHAEL T. SWANSTON \\ Dundee College of Technology, Dundee, Scotland \\ and \\ NICHOLAS J. WADE \\ Dundee University, Dundee, Scotland
}

\begin{abstract}
If physical movements are to be seen veridically, it is necessary to distinguish between displacements over the retina due to self-motion and those due to object motion. When target motion is in a different direction from that of a pursuit eye movement, the perceived motion of the target is known to be shifted in direction toward the retinal path, indicating a partial failure of compensation for eye movements (Becklen, Wallach, \& Nitzberg, 1984). The experiments reported here compared the perception of target motion when the head and/or eyes were moving in a direction different from that of the target. In three experiments, target motion was varied in direction, phase, and extent with respect to pursuit movements. In all cases, the compensation was less effective for head than for eye movements, although this difference was least when the extent of the tracked and target motions was the same. Compensation for pursuit eye movements was better than that reported in previous studies.
\end{abstract}

Human visual perception normally occurs during the course of complex movements of the eyes and of the body. How objects in the environment appear to move will therefore depend on a separation of the displacement of the retinal image due to such self-motions from that due to object movement. Compensation for eye movements is necessary for stability of the visual world, and has been extensively discussed (e.g., Mack, 1986; Post \& Leibowitz, 1985; Swanston, Wade, \& Day, 1987; von Holst, 1954). While, in general, such compensation is effective, differences in motion perception can occur when the eyes are moving as opposed to when they are stationary. In the simplest case, a moving target may be tracked by pursuit eye movements, or observed during fixation of a stationary point. The velocity of tracked movement is underestimated (Mack \& Herman, 1973, 1978), a phenomenon known as the Aubert-Fleischl paradox. If a stationary background is present during the tracking of a target, it may appear to move in the direction opposite to that of the target; this is the Filehne illusion (Mack \& Herman, 1973). Both these effects are relatively slight, indicating that compensation for eye movements is generally effective with respect to a tracked target or a stationary background. However, if there is a second moving visual target, its apparent direction of motion is displaced toward the retinal path produced by the objective motion and the eye movement (Becklen, Wallach, \&

The authors would like to thank Arien Mack and an anonymous referee for their helpful comments on an earlier version of this paper. M. T. Swanston's mailing address is Dundee College of Technology, Bell Street, Dundee DD1 1HG, Scotland. N. J. Wade's address is Department of Psychology, Dundee University Dundee DD1 4HN, Scotland.
Nitzberg, 1984; Festinger, Sedgwick, \& Holtzman, 1976; Wallach, Becklen, \& Nitzberg, 1985). Becklen et al. suggested that the normal correction of retinal motion by eyemovement information (as occurs for the tracked target) does not apply to the motion of a target moving at an angle to the direction of the eye movement. If so, this would suggest that the compensatory process is orientation selective, in the sense that it is effective only if the eye movement and the retinal path are in the same direction. Wallach et al. argued that the system is unable to perform a vector analysis of retinal motion, which would be required in order to separate out the effects of a physical movement from those of an eye movement in a differing direction. If so, then compensation should not vary with the relative orientation between the target's retinal path and the eye movement.

While there has been considerable investigation of the processes responsible for relating eye-movement information to retinal-image motion, much less is known about other types of observer motion. This is so despite the fact that observers are seldom stationary, and that the consequences of head and body motion pose at least as great a perceptual problem as do those of eye movements. Thus, the purpose of the experiments reported here was to examine movement perception when equivalent displacements of the retinal image resulted from movements not only of a visual object, but also of the eyes and of the head.

\section{EXPERIMENT 1}

In two of the conditions of this experiment, observers moved either eyes or head to track a horizontally moving 
target. At the same time, they judged the apparent direction of motion of a second test target moving at various angles to the horizontal, the displacement of which was approximately one-third of that through which the head or eyes moved. The resulting retinal path could be calculated, and compared with the reported direction of motion, for a range of physical directions. While Becklen et al. (1984) and Wallach et al. (1985) compared vertical and horizontal motion of the target during horizontal eye movements, they did not study intermediate directions, and no information is available regarding the comparative effects of eye and head movements. In two control conditions of the present experiment, the tracked target was stationary and centrally located. Observers fixated this target either with head stationary or with lateral head movements.

\section{Method}

Observers. Eight observers took part in the experiment. They were paid volunteers, and all had normal or corrected-to-normal vision.

Apparatus. Displays were presented on a 35 -cm-diagonal video monitor (CBM 1701), under the control of a microcomputer (CBM 64), with a viewing distance of $70 \mathrm{~cm}$. Observations were made in darkness, and only the targets on the screen were visible. The contrast of the targets was reduced so that no visible trace was left during a movement. The targets were two dots, subtending approximately $0.05^{\circ}$ in diameter. One, the tracked target, could move through a horizontal distance of $9.7^{\circ}(120 \mathrm{~mm}$ on the screen); when stationary, it was presented in the center of this range. The test target was located to the left of the first, and could move along a path inclined at various angles to the horizontal. These motion paths were along diameters of a circle whose center was aligned horizontally with the tracked target and located $2.5^{\circ}$ to the left of the leftmost position of the tracked target when it moved. Each path subtended $2.9^{\circ}$ (36 mm on the screen). The observer's head was placed on a chinrest, which was mounted on runners and could move left and right through a distance of $120 \mathrm{~mm}$. The center of the head movement was aligned with the central position of the tracked target. Lateral excursions of the observer's head were detected by a position transducer, whose output was read by the microcomputer that generated the display. This value was used to index calibrated lookup tables for positions of the tracked and test targets, so that the motion of either target could be made dependent on movements of the head. There was no appreciable lag in the response time of the system, since the program was written in machine code. Resolution of head position was approximately $1 \mathrm{~mm}$.

Retinal paths. The retinal paths traced by the test target in each condition can be calculated if fixation on the tracked target is assumed to have been accurate. Figure 1 illustrates the physical and retinal motions for vertical motion of the test target in each condition. In Condition 1, the retinal path for each direction of motion was the same as the physical direction. In Conditions 2 and 3 , the movement of the observer's eyes or head, respectively, added a horizontal vector to the motion of the test target on the retina. This was in the same direction as any real vector of horizontal motion of the test target, the magnitude of which depended on the physical direction. Thus, if the test target moved vertically, there was no real horizontal component, and the retinal path was the result of adding a horizontal vector due to eye or head displacement. If the motion was horizontal, no change in the orientation of the retinal path took place, but its length was increased. In general, for the phase relationships used in this experiment, the retinal path angle $(p)$ in degrees from the vertical for Conditions 2 and 3 is given by

$$
\tan p=M+d \cdot \sin (t) / d \cdot \cos (t),
$$

where $d$ is the physical length of the path traversed by the test target, $t$ is the angle between this path and the vertical, and $M$ is the length of the path traversed by the tracked target (or the extent of head movement in Condition 3). In the discussion of this and subsequent experiments, motion-path angles are expressed with respect to the vertical, with angles clockwise from vertical being positive, and vice versa. The retinal path in Condition 4 did not deviate significantly from the physical path. Table 1 includes the retinal path angles for each condition and direction of physical motion. All values in this experiment were positive.

Procedure. Observations were binocular, and were made under four conditions. In Condition 1, the head was kept still in the central position and the observer fixated the tracked target, which was also stationary and straight ahead. In Condition 2 , the head was still, but the tracked target moved back and forth horizontally at a velocity of $4.5^{\circ}$ per second (dps) and was pursued by eye movements. For Condition 3, observers moved their head from side to side and the tracked target was moved concomitantly on the screen so as to remain straight ahead. They were instructed to move at a steady pace, so that a complete left-to-right excursion (or vice versa) took about $2 \mathrm{sec}$. Practice in this was given before the start of the experiment, and none of the observers had any difficulty with the task. Similarly, none reported any problem in maintaining fixation on the tracked target, or in forming a clear impression of the direction of motion of the test target. Subjectively, it appeared that the tracked target was moving independently so as to remain in the

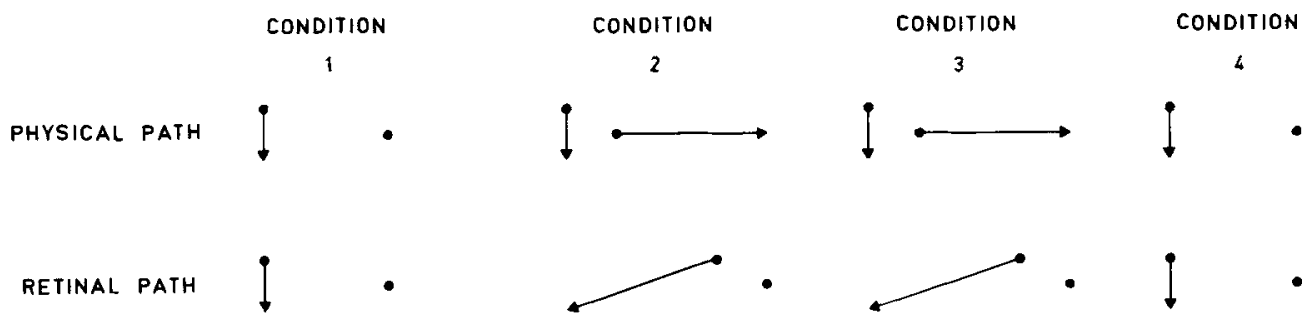

Figure 1. Schematic representation of stimuli employed in Experiment 1. Diagrams represent physical and retinal motion paths in the four conditions (Condition 1-stationary eyes and head; Condition 2 -eyes moving, head stationary; Condition 3-eyes stationary, head moving; Condition 4-eyes and head moving), for vertical motion of the test target. In Conditions 2 and 3 , the retinal path was displaced as a result of eye or head movement. Arrows (not present in the actual display) represent the initial concurrent directions of motion of the tracked and test targets. On reaching the limit of travel, both directions were reversed, and this cycle was repeated. 
straight ahead position; there was no impression of driving the target by means of head movements. In Condition 4 , the same head movements were made, but the tracked target remained stationary in the central position. In all conditions, the observers were instructed to maintain fixation on this target. The test target moved during the trial along one of seven paths that varied between vertical and horizontal, in steps of $15^{\circ}$. In Conditions 2 and 3, the test target moved down and/or to the left as the tracked target moved to the right. Observers were instructed to continue until they had judged the direction of motion of the target (usually after 5 to 10 cycles). They gave a verbal indication when this was completed, and a light was switched on in the observation booth. To the left of the chinrest, at eye height, a metal rod, $12 \mathrm{~cm}$ in length, was mounted on a potentiometer facing the observer. The position of this rod was adjusted so that its angle to the vertical matched that of the perceived motion path of the test target, and the setting was recorded. Three repetitions of this procedure for each motion direction were made for each condition, in a random order.

\section{Results}

Table 1 summarizes the results of the experiment. The physical and retinal paths are shown for each condition, together with the mean apparent direction of motion of the test target in degrees from the vertical. It can be seen that vertical and horizontal motions were judged correctly in Condition 1 (static observation), but that intermediate values showed an overestimation of the steepness of the path, which was most marked at $45^{\circ}$. This bias in the judgment of oblique motion paths has also been reported by Post and Chaderjian (1987), under similar conditions. A comparison of Condition 4 with Condition 1 showed that there was unlikely to have been an error in the perceived distance of the targets. This could have produced an effect on the motion path perceived with head movements, but not with eye movements, and would therefore have been a confounding factor. It has been shown in a number of studies that the motion of a stimulus viewed during a lateral head movement is related to its perceived egocentric distance (e.g., Gogel, 1982; Gogel \& Tietz, 1974). Thus, if a stimulus is seen as more distant than its physical distance, it will appear to move against the direction of concomitant lateral head movement, and vice versa (see Swanston et al., 1987, Figure 5). In principle, an error in perceived distance could therefore have produced a larger difference in the displacement of the perceived motion path with concomitant head movements as opposed to eye movements. Since the data for Conditions 1 and 4 were not significantly different across motion-path directions $[F(1,7)=1.76]$, it can be concluded that the perceived distance of the target was approximately veridical, presumably due to the availability of accommodation and binocular convergence as cues to distance. The data from Condition $\mathbf{4}$ also indicate correct registration of head movements, since if this had been in error, there would have been a distortion of the perceived path of the tracked target relative to that obtained with static observation. However, this should probably not be generalized to the other head-movement condition (Condition 3), since in the latter case the eyes were still, whereas in Condition 4 they were rotated so that fixation on the stationary central target could be maintained. This additional eye-movement information may have contributed to the perception of head movement.

The extent of the perceived deviation from a physically vertical path was about $11^{\circ}$ with eye movements and about $27^{\circ}$ with head movements. For both there was a gradual decline as the physical direction of the motion approached horizontal. This pattern of results might appear to support the conclusion that compensation for head or eye movements improved as the direction of tracked and test motion became more similar. However, this conclusion may not be justified, inasmuch as there was an increasing physical vector of horizontal motion in the test target as its path approached horizontal. Thus, a constant level of compensation would have produced a declining deviation of the perceived path from the physical path.

One way to examine this issue is to calculate the extent of the compensation as a percentage of the horizontal retinal displacement due to eye or head movement. For each subject, a compensation score was derived by comparing the horizontal vectors under different conditions. First, the difference was obtained between the horizontal vector of the perceived path during eye or head movement and that during static observation; the latter was used to allow for the systematic biases in reports of motion direction described above. The compensation score is the difference between the physical extent of the horizontal vector due to eye or head movement and the above value, expressed as a percentage of this horizontal vector. Table 1 shows the mean compensation values for eye move-

Table 1

Means and Standard Errors of Perceived Motion Paths for Each Condition and Physical Motion Path, and Mean Compensation for Eye and Head Movements, in Experiment 1

\begin{tabular}{|c|c|c|c|c|c|c|c|c|c|c|c|c|c|}
\hline \multirow{2}{*}{\multicolumn{2}{|c|}{$\begin{array}{c}\text { Motion Path: } \\
\text { Conditions } 2 \text { and } 3 \\
\end{array}$}} & \multicolumn{8}{|c|}{ Perceived Path (in degrees) } & \multicolumn{4}{|c|}{ Compensation (\%) } \\
\hline & & \multicolumn{2}{|c|}{ Condition 1} & \multicolumn{2}{|c|}{ Condition 2} & \multicolumn{2}{|c|}{ Condition 3} & \multicolumn{2}{|c|}{ Condition 4} & \multicolumn{2}{|c|}{ Condition 2} & \multicolumn{2}{|c|}{ Condition 3} \\
\hline Physical & Retinal & $M$ & $\overline{S E}$ & $M$ & $\overline{S E}$ & $M$ & $\overline{S E}$ & $M$ & $S E$ & $M$ & $S E$ & $M$ & $S E$ \\
\hline 0 & 73.3 & -0.9 & 0.7 & 11.1 & 2.8 & 26.8 & 3.5 & -0.9 & 1.9 & 93.5 & 1.5 & 83.6 & 2.9 \\
\hline 15 & 75.0 & 9.1 & 0.8 & 15.0 & 2.2 & 29.6 & 3.9 & 8.3 & 2.3 & 96.7 & 1.2 & 86.8 & 3.5 \\
\hline 30 & 77.3 & 22.0 & 2.3 & 25.7 & 3.0 & 39.0 & 3.7 & 20.1 & 1.4 & 97.4 & 2.9 & 86.4 & 6.2 \\
\hline 45 & 80.1 & 30.3 & 1.2 & 36.9 & 3.9 & 48.1 & 3.7 & 31.0 & 1.9 & 94.3 & 3.6 & 82.8 & 6.4 \\
\hline 60 & 83.2 & 50.4 & 2.4 & 54.0 & 4.7 & 59.2 & 3.6 & 45.0 & 3.3 & 98.6 & 3.1 & 87.7 & 5.4 \\
\hline 75 & 86.0 & 70.4 & 1.6 & 73.5 & 3.7 & 78.5 & 2.2 & 66.9 & 2.4 & 91.6 & 5.2 & 73.5 & 9.4 \\
\hline 90 & 90.0 & 89.8 & 0.4 & 90.1 & 0.2 & 89.7 & 0.2 & 89.0 & 0.7 & & & & \\
\hline
\end{tabular}


ment and head movement for physical paths between vertical and $75^{\circ}$.

Analysis of variance of the compensation values showed that there was no significant effect of the physical path orientation $[F(5,35)=1.35]$, although the difference between head and eye movement was significant $[F(1,35)$ $=9.33, p<.05]$. The mean compensation values were $95.1 \%$ for eye movement and $83.6 \%$ for head movement. The value for eye-movement compensation was higher than that reported by Festinger et al. (1976) or Becklen et al. (1984). However, in both these studies, the observation conditions were not wholly comparable to the present case, since the configuration of the test and tracked targets may have itself contributed to the perceived motion path. In the Festinger study, the display contained an additional dot whose position was adjusted to record the target motion path. Becklen et al. examined only vertical motion of a test target during horizontal tracking, but the paths of the tracked and test targets intersected. It is therefore likely that part of the perceived shift in the direction of motion was due to the interaction between simultaneously presented movements in differing directions that can occur with or without eye movements (e.g., Gogel, 1974; Johansson, 1958). Becklen et al. (1984, Experiment 2) found that the contribution of such configurtional change was around $25 \%$ of the observed tilt of a vertical path. On the basis of the present data, although compensation for eye movements is not complete, the perceived path is much closer to the physical path than to the retinal path. It could be argued that the apparently greater compensation for eye movements obtained here was due to a failure to track the target motion accurately; in this case, the retinal path would deviate less from the physical path. Although recordings of observers' eye movements were not made, the velocity of the tracked target ( $4.5 \mathrm{dps})$ was well within the range that can be followed accurately by pursuit eye movements (Howard, 1982). Ocular pursuit by observers in this experiment would have had to be very poor to account for the improvement in compensation relative to that obtained in previous studies. The experiments reported below were designed to investigate other possible causes of this difference, and to examine further the difference in the compensation of head and eye movements.

\section{EXPERIMENT 2}

Although the physical motion paths in Experiment 1 varied through $90^{\circ}$, between vertical and horizontal, the corresponding retinal paths covered a much more restricted range of about $16^{\circ}$. This was due to the phase relationship between the motions of the two targets, since the fixation target moved from right to left as the test target moved down and/or to the left. In Experiment 2, the direction and phase of the test target with respect to the tracked target was varied so as to give a range of variation in the retinal path of around $160^{\circ}$. The purpose of this was to examine the relative effectiveness of compensation for eye and head movements with larger discrepancies between physical and retinal motion.

\section{Method}

Observers. Eight observers took part in the experiment; 5 of them had participated in Experiment 1. All had normal or corrected-tonormal vision.

Apparatus. The apparatus was the same as that employed in Experiment 1.

Procedure. The same four observation conditions were used as in Experiment 1. The extents and locations of the movements of both the tracked and test targets were also the same. The test target moved along one of two paths, whose right-hand limit of motion was either $45^{\circ}$ to the right or $45^{\circ}$ to the left of vertical, designated +45 and -45 , respectively (see Figure 2 ). In addition, the phase of each of these motions was varied with respect to the tracked target. Thus, as the tracked target moved from left to right (autonomously in Condition 2 and concomitantly with the observer's head in Condition 3), the tracked target moved on either the upward or downward phase of its path. This meant that the horizontal vector of retinal motion produced by observer motion was either in the same direction as the horizontal vector of the physical motion or opposed to it. In Conditions 2 and 3, for each physical path there were therefore two retinal paths, giving a total of four stimulus conditions, with retinal paths of $+80.1^{\circ},-74.9^{\circ},-80.1^{\circ}$, and $+74.9^{\circ}$, respectively. Path 1 replicated the correspondingly oriented stimulus in Experiment 1. In Conditions 1 and 4, these physical paths were also the retinal paths, within the limits of accurate fixation. Figure 2 illustrates the physical and retinal paths for Conditions 2 and 3.

\section{Results}

As before, Condition 1 provided a control for judgments of the direction of a motion path, in the absence

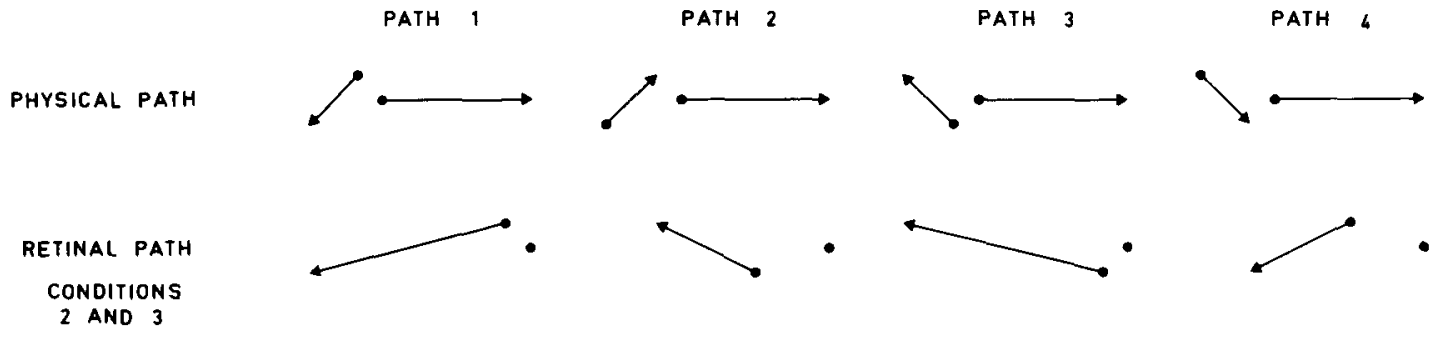

Figure 2. Schematic representation of the stimuli employed in Experiment 2 (diagrammatic conventions are as in Figure 1). 
Table 2

Means and Standard Errors of Perceived Motion Paths for Each Condition and Physical Motion Path, and Mean Compensation for Eye and Head Movements, in Experiment 2

\begin{tabular}{|c|c|c|c|c|c|c|c|c|c|c|c|c|c|c|}
\hline \multirow{2}{*}{\multicolumn{3}{|c|}{$\begin{array}{c}\text { Motion Path: } \\
\text { Conditions } 2 \text { and } 3\end{array}$}} & \multicolumn{8}{|c|}{ Perceived Path (in degrees) } & \multicolumn{4}{|c|}{ Compensation (\%) } \\
\hline & & & \multicolumn{2}{|c|}{ Condition 1} & \multicolumn{2}{|c|}{ Condition 2} & \multicolumn{2}{|c|}{ Condition 3} & \multicolumn{2}{|c|}{ Condition 4} & \multicolumn{2}{|c|}{ Condition 2} & \multicolumn{2}{|c|}{ Condition 3} \\
\hline Path & Physical & Retinal & $M$ & $S E$ & $M$ & $S E$ & $M$ & $S E$ & $M$ & $S E$ & $M$ & $S E$ & $M$ & $S E$ \\
\hline 1 & +45 & +80.1 & +30.6 & 2.3 & +32.8 & 3.1 & +49.0 & 5.6 & +33.0 & 4.6 & 97.2 & 1.1 & 76.7 & 9.2 \\
\hline 2 & +45 & -74.9 & +28.1 & 1.7 & +16.8 & 1.9 & -21.9 & 8.3 & +29.9 & 1.9 & 93.9 & 1.4 & 69.3 & 9.5 \\
\hline 3 & -45 & -80.1 & -38.1 & 2.7 & -39.5 & 5.2 & -55.0 & 4.7 & -42.1 & 2.9 & 95.8 & 5.5 & 78.1 & 9.2 \\
\hline 4 & -45 & +74.9 & -39.7 & 1.9 & -21.1 & 3.6 & +19.1 & 5.4 & -35.6 & 2.3 & 88.8 & 1.7 & 72.3 & 2.9 \\
\hline
\end{tabular}

Note-See text and Figure 2. Values are in degrees from vertical (+ve, clockwise; -ve, counterclockwise).

of observer movement. Table 2 shows the physical and retinal paths for each condition, and the mean perceived path angle. First, it can be noted that the judgments in Condition 1 again showed a tendency toward "steepness." Path 1 was judged as $+30.6^{\circ}$ from the vertical, which is very close to the value of $+30.3^{\circ}$ with the same stimulus in Experiment 1. Similarly, Path 2, which was also physically at $+45^{\circ}$, was judged as $+28.1^{\circ}$. However, this tendency was less marked with paths oriented counterclockwise from vertical; Path 3 was judged as $-38.1^{\circ}$, and Path 4 as $-39.7^{\circ}$. The origin of these deviations was not of direct interest in these experiments, but the values obtained provide a baseline for evaluating judgments during observer movement.

There was a marked difference between the magnitudes of the effects of head and eye movements, although the pattern of results was the same in each case. In Condition 2, Paths 1 and 3 showed only a small deviation from the physical direction; in fact, the values were of the same order as those obtained in Condition 4. However, Paths 2 and 4 indicated much larger shifts in the perceived direction of motion, by $11.3^{\circ}$ and $18.6^{\circ}$, respectively, relative to the equivalent perceived paths in Condition 1. In these cases, the effect of an eye movement was to produce a larger difference between the physical and retinal paths. Similar relationships applied in Condition 3, except that the perceived paths were more displaced from the physical paths for all motion paths. Thus, Paths 1 and 3 were $+18.4^{\circ}$ and $-16.9^{\circ}$ different from those of Condition 1 , respectively. The value for Path 1 was close to that obtained in Experiment $1\left(17.8^{\circ}\right)$. Paths 2 and 4 gave values of $-50.0^{\circ}$ and $58.8^{\circ}$ when compared with those of Condition 1 . These large changes from the path perceived by a stationary observer were in the direction predicted from the retinal path, and were clearly larger when the potential displacement was larger. As a result, as shown in Table 2 , the percentage compensation for the horizontal vector due to head or eye movement was approximately constant in each case. The mean compensation was $74.1 \%$ for head movement and $93.9 \%$ for eye movement. Analysis of variance showed that there was a significant difference in the compensation for head and eye movement $[F(1,7)=7.73, p<.05]$, but none due to the different retinal paths $[F(3,21)=1.0]$ and no interaction $[F(3,21)$ $=0.7]$.

\section{EXPERIMENT 3}

In the two previous experiments, the tracked target moved through a distance approximately three times that of the test target. The resulting horizontal vector applied to the retinal motion of the test target was therefore relatively large, and the displacements of this path from the physical value were correspondingly great. Experiment 3 examined the relationship between the effectiveness of compensation for eye or head movements and the extent of the vector to be compensated for.

\section{Method}

Observers. Twelve observers took part in the experiment. Three had participated in both previous experiments, and 1 had participated in Experiment 2. All had normal or corrected-to-normal vision.

Apparatus. The apparatus was the same as that used in the previous two experiments.

Procedure. The motion path of the test target was always vertical. However, the downward phase of this motion was synchronized with either the rightward or the leftward phase of the motion of the tracked target. The horizontal vector of retinal motion due to tracking therefore produced a retinal path tilted either clockwise or counterclockwise from the vertical, respectively. There were three extents of movement of the tracked target $(40,80$, and $120 \mathrm{~mm})$, giving three retinal paths on each side of the vertical, at $\pm 48.0^{\circ}$, $\pm 65.8^{\circ}$, and $\pm 73.3^{\circ}$. Each target was observed with eye movement and with head movement. In addition, the vertical motion of the test target was presented to a stationary observer fixating a point in the center of the range of the tracked target, as a control. The conditions are illustrated schematically in Figure 3. Each condition was seen four times by each observer.

\section{Results}

Judgments of the direction of motion of the test target in the various conditions were used to compute the mean percentage compensation for the horizontal vector due to eye or head motion. Data for equivalent clockwise and counterclockwise retinal paths were combined, and the results are shown graphically in Figure 4. Analysis of variance showed that the main effects of tracking type (by head or eye movements) and extent of observer motion were significant $[F(1,11)=17.0, p<.01$, and $F(2,11)$ $=13.8, p<.01$, respectively], although the interaction was not $[F(2,11)=1.17]$. The difference between compensation for head and eye movement with the largest extent of movement replicated the finding with the same 
$1: 1$

PHYSICAL PATH

RETINAL PATH

RETINAL PATH
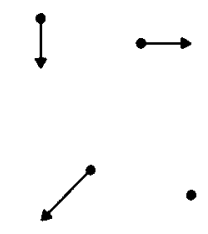

$\bullet$
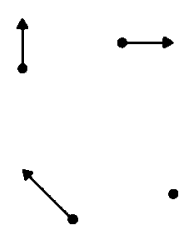

$1: 2$
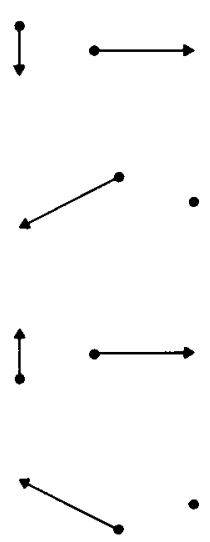

$1: 3$
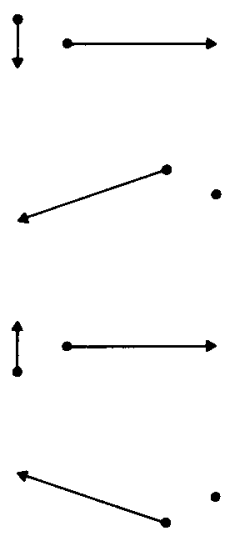

Figure 3. Schematic representation of the stimuli employed in Experiment 3 (diagrammatic conventions are as in Figure 1).

parameters in Experiment 1, although the perceived paths were somewhat closer to the retinal path than was found earlier. In addition, this experiment showed that compensation was reduced as the extent of eye or head motion was reduced. This is of particular interest, since it suggests a reason for the higher compensation for eye movements obtained in these experiments than in previous studies. For example, Becklen et al. (1984) employed approximately equal values for the extent of the test and tracked motions in compensation for eye movements. Their Experiment 2, which can therefore be compared to the equivalent condition of the present experiment, gave a compensation value of around $35 \%$, based on the figures given for the perceived tilts and extent of eye movements. However, if allowance is made for their estimate that $8.4^{\circ}$ of the tilt was produced by configurational changes, independent of eye movements, then the compensation was approximately $54 \%$, a value reasonably comparable to the $68 \%$ obtained here. In addition, the data from this experiment suggest that as the extent of the horizontal motion vector was increased in comparison with the physical extent of the test motion, compensation increased by less for head movements than for eye movements. If so, then, experimental comparison of compensation for eye or head movements must take account of the extent of the motion; the two processes may be more similar in effectiveness when the test and tracked motions are approximately equal in extent.

\section{EXPERIMENT 4}

As discussed above, when two or more visual objects undergo simultaneous motion in differing directions, their perceived paths differ from when they are seen in isola-

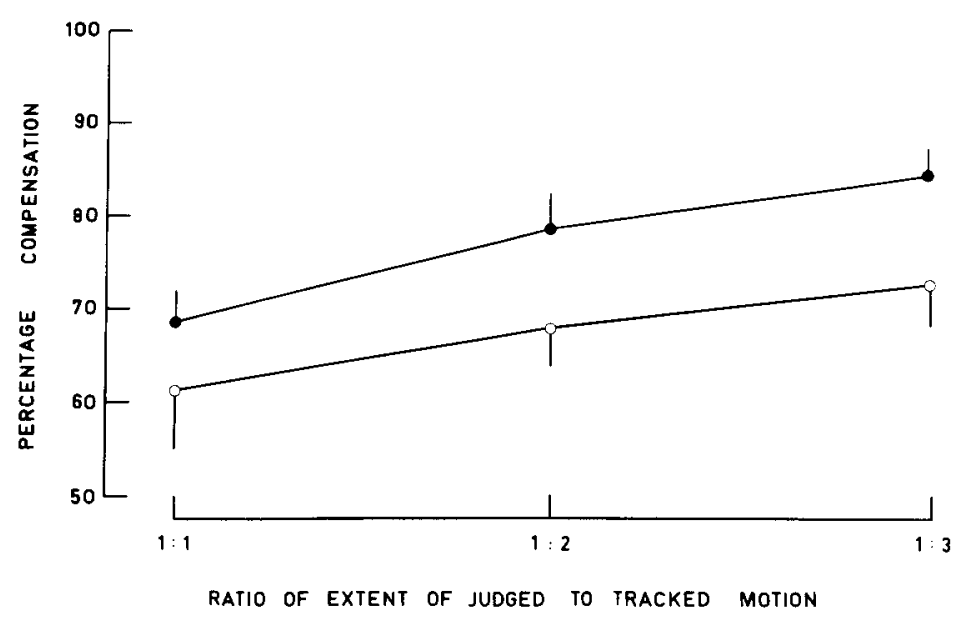

Figure 4. Graph showing the results of Experiment 3. Mean compensation for eye (filled circles) and head movements (open circles) are shown as a function of the ratio of the extents of the test and tracked motions. Vertical bars represent one standard error of the means. 
tion. Such motion interaction has been termed a configurational effect, and there is extensive empirical evidence for its occurrence and characteristics (see Wade \& Swanston, 1987 , for a review). One well-established fact is that configurational effects depend upon the adjacency of the moving objects; the interaction of their perceived motions declines with separation over a few degrees (Gogel, 1974). The display for the present experiments was designed to have the maximum separation between the tracked and test targets. Clearly, any influence of configurational change would be confounded with the compensation for eye or head movements, which was the main concern. During a trial, the motions of each target meant that the separation was changing, so that they were closer when the tracked target was at the left end of its range. The mean separation was approximately $7.5^{\circ}$, with a range of $2.5^{\circ}$ to $12.5^{\circ}$. Experiment 4 was designed to assess the effect of motion interaction due to configurational changes, that might have influenced the results of the previous experiments.

\section{Method}

Observers. Ten observers took part in the experiment. All had normal or corrected-to-normal vision, and 5 had participated in one of the previous experiments.

Apparatus. The apparatus was the same as that employed in the previous experiments.

Procedure. The display and the conditions of observation were similar to those used with vertical target motion in Experiment 1. However, an additional stationary target was presented in the center of the path of the tracked target. Three of the observation conditions were the same as those in Experiment 1. In Condition 1, observers fixated the central stationary target and there was no moving tracked target. In Condition 2, the tracked target was pursued by eye movements, and in Condition 3 , it was pursued by head movements. For Condition 4, the tracked target moved horizontally, as in Condition 2, but observers fixated the central stationary target. In all conditions, the observers' task was to judge the perceived direction of motion of the test target. Each condition was seen four times, in a random order.

\section{Results}

The essential comparison of the experiment was between Conditions 1 and 4 . A configurational effect would be shown by a deviation in the perceived path of the test target when it was seen in conjunction with a horizontally moving target, with the eyes and head stationary. The mean perceived paths in these two conditions were identical, at $2.7^{\circ}$ counterclockwise. There was therefore no indication that the perceived tilts measured in the previous experiments were altered by an interaction between the motions of the test and tracked targets. The data for Conditions 2 and 3 provided a test of whether the addition of a third, stationary, target influenced the effects of eye or head movements. The mean perceived path was $8.2^{\circ}$ clockwise for Condition 2 and $13.6^{\circ}$ clockwise for Condition 3 . These values were comparable to those obtained earlier, although the deviation from the physical path was perhaps somewhat reduced. It is possible that the static target may have provided additional information for eye or head movements. The difference between the two conditions was in the same direction as before, and was significant $[t(9)=5.1, p<.01]$.

\section{DISCUSSION}

The experiments described above were concerned with the perception of object motion during movements of the eyes and of the head. If such movements are in a direction different from that of an observed object, then motion of the object's image over the retina will be the result both of its own motion and of the observer's movement. The direction of image motion will therefore differ from the physical direction. Accordingly, the main purpose of these experiments was to compare perceived direction when displacements of the retinal image were due to pursuit eye movements and when they were due to pursuit head movements. In the former case, the head was stationary; in the latter, the eyes were stationary, within the limits of accurate pursuit. However, to a close approximation, the effects on the retinal image were the same. The similarity of the perceived path to the physical path provided a measure of the ability to take account of head or eye movements in recovering the veridical direction of object motion. In all four experiments it was found that compensation was significantly better for eye movements than for equivalent head movements. Compensation for eye movements was generally better than had been found in previous studies (Becklen et al., 1984; Festinger et al., 1976), although Experiment 3 showed that this may have been due to the extent of the tracked movement. In Experiment 1 , object motion varied between vertical and horizontal, with horizontal eye or head movement. The percentage compensation for this horizontal vector remained approximately constant. When much larger shifts in retinal motion were introduced in Experiment 2, by varying the phase relationships between the object motion and the pursuit motion, compensations for head and eye movements were again significantly different, but in each case a constant percentage of the horizontal vector was compensated for. Experiment 3 showed that the difference between head- and eye-movement compensation was a function of the magnitude of the retinal vector due to pursuit; this difference was reduced when the pursuit movement was equal in extent to the object motion. A possible explanation for this is that information regarding larger head movements is less accurate, although this remains to be tested. In general, the experiments confirm the earlier findings of a failure to compensate fully for pursuit eye movements when the object motion is in a different direction, although the results show a greater degree of compensation than has been reported before; the perceived path was always closer to the physical path than to the retinal path. Similarly, it has been shown that 
there is a failure to compensate fully for retinal image displacements due to head movements, and that this is a larger effect.

These results conform to the model of motion perception proposed by Swanston et al. (1987). This model defines the logical steps needed to reach a representation of the movement of objects in the environment independent of any self-movement. Information regarding retinal motion, eye movements, head or body movements, and perceived egocentric distance are combined to reach what is termed a "geocentric" representation of object movement-that is, a perception of motion with respect to three-dimensional space. The present experiments suggest that the visual system falls short of ideal performance in this respect, in that the consequences of eye or head movement cannot be completely separated from object movement, especially when these are in different directions. One aspect of the model not tested here is that although eye movements can be directly related to retinal image motion, this is not possible for self-movement. The reason for this is that eye movements and the consequent retinal image displacements are independent of the perceived distance of a visual object; in effect, both can be expressed in terms of angular direction. Selfmovements, on the other hand, are necessarily threedimensional, and compensation for their effects on the retinal image would have to be in terms of a similar threedimensional representation of object movement. Thus, the perceived egocentric distance of an object would interact with compensation for head movement but not with compensation for eye movement. Control conditions in the present experiments showed that perceived distance was not systematically different from physical distance, so that this factor did not influence the relative effects of head and eye movements. Further comparison of motion perception during head and eye movements, employing variation in perceived distance, will enable this prediction to be tested.

\section{REFERENCES}

Becklen, R., WAllach, H., \& Nitzberg, D. (1984). A limitation of position constancy. Perception \& Psychophysics, 10, 713-723.

Festinger, L., Sedgwick, H. A., \& Holtzman, J. D. (1976). Visual perception during smooth pursuit eye movements. Vision Research, 16, $1377-1386$.

GOGEL, W. C. (1974). Relative motion and the adjacency principle. Perception \& Psychophysics, 26, 425-437.

GoGEL, W. C. (1982). Analysis of the perception of motion concomitant with a lateral motion of the head. Perception \& Psychophysics, 32, 241-250.

GOGEL, W. C., \& TIETZ, J. D. (1974). The effect of perceived distance on perceived movement. Perception \& Psychophysics, 16, 70-78.

HowARD, I. P. (1982). Human visual orientation. New York: Wiley. Johansson, G. (1958). Rigidity, stability and motion in perceptual space. Acta Psychologica, 14, 359-370.

MACK, A. (1986). Perceptual aspects of motion in the frontal plane. In K. R. Boff, L. Kaufman, \& J. P. Thomas (Eds.), Handbook of perception and human performance (Vol. 1, chap. 17, pp. 1-38). New York: Wiley.

Mack, A., \& Herman, E. (1973). Position constancy during pursuit eye movements: An investigation of the Filehne illusion. Quarterly Journal of Experimental Psychology, 25, 71-84.

MaCK, A., \&erman, E. (1978). The loss of position constancy during pursuit eye movements. Vision Research, 18, 55-62.

Post, R. B., \& Chaderian, M. (1987). Perceived path of oblique motion. Perception, 16, 23-28.

Post, R. B., Leirowitz, H. W. (1985). A revised analysis of the role of efference in motion perception. Perception, 14, 631-643.

Swanston, M. T., WAdE, N. J., \& DAY, R. H. (1987). The representation of uniform motion in vision. Perception, 16, 143-159.

voN HoLST, E. (1954). Relations between the central nervous system and the peripheral organs. British Journal of Animal Behaviour, 2, 89-94.

WADE, N. J., \& SWANSTON, M. T. (1987). The representation of nonuniform motion: Induced movement. Perception, 16, 555-571.

Wallach, H., Becklen, R., \& NitzBerg, D. (1985). The perception of motion during colinear eye movements. Perception \& Psychophysics, 38, 18-22.

(Manuscript received March 4, 1987; revision accepted for publication November $25,1987$. ) 